\title{
A Case of Fungal Keratitis Secondary to Cylindrocarpon Destructans*
}

\author{
Rajen Tailor $^{1 \#}$, Ibrahim Elaraoud ${ }^{2}$, Mike Quinlan ${ }^{3}$ \\ ${ }^{1}$ Birmingham and Midlands Eye Centre, City Hospital, Birmingham, England; ${ }^{2}$ University Hospitals of Coventry and Warwick, \\ Coventry, England; ${ }^{3}$ Russells Hall Hospital, Dudley, England. \\ Email: " rajentailor@doctors.org.uk
}

Received December $23^{\text {rd }}$, 2011; revised January $28^{\text {th }}$, 2012; accepted February $18^{\text {th }}$, 2012

\begin{abstract}
Purpose: To report the first human case of fungal keratitis caused by Cylindrocarpon destructans and to highlight the issues with the use of topical steroids, the duration of antifungal treatment and the potential role of topical ciclosporin. Methods: A patient presented following being injured in the left eye by a fuchsia plant. Data was collected by slit lamp examination and review of the case notes and microbiology reports. Results: No organisms were cultured from a corneal scrape however cultures from a corneal biopsy identified cylindrocarpon species morphologically resembling Cylindrocarpon destructans. The patient responded well to topical amphotericin and clotrimazole and oral voriconazole but, developed a corneal perforation, which required an urgent tectonic penetrating keratoplasty (PKP). Despite being on topical dexamethasone and natamycin, the patient presented two months post-operatively with a corneal epithelial defect and a large hypopyon. Subsequently, the patient developed a deep corneal infiltrate and corneal vascularisation with a persistent epithelial defect. Conclusion: This is the first reported case of keratitis caused by Cylindrocarpon destructans. The case highlights: the contentious issues in the use of topical steroids following PKP and the duration of antifungal treatment both in primary infection and following PKP. Furthermore, the case accentuates a potential role for ciclosporin as an alternative to steroids following PKP.
\end{abstract}

Keywords: Cylindrocarpon Destructans; Ciclosporin; Fungal Keratitis; Penetrating Keratoplasty; Topical Steroids

\section{Case}

A 74-year-old man sustained an accidental injury to the left eye (OS) by a fuchsia plant. The following day he attended the local emergency department complaining of redness and soreness. His visual acuity in the left eye was noted to be 6/9 and a large corneal abrasion was identified. He was commenced on chloramphenicol ointment four times a day and referred to the eye clinic.

On review in the eye clinic, the patient complained of severe eye pain. His visual acuity (VA) was $6 / 30$ OS and slit lamp biomicroscopy examination showed a large epithelial defect, 2+ of anterior chamber white cells and iris hemorrhages. He was commenced on guttae (G) cefuroxime $5 \%$ and ofloxacin $0.3 \%$ hourly. The patients symptoms gradually improved and on day seven of topical antibiotic treatment, prednisolone 0.5\% QDS was added. Three days later, the patient presented with worsening of his symptoms associated with a $3.6 \mathrm{~mm}$ hemorrhagic hypopyon (Figure 1). The patient was admitted to

\footnotetext{
*Disclaimer: No proprietary interest. No financial support was received.

${ }^{\#}$ Corresponding author.
}

the eye ward where all treatment was stopped for 24 hours and a corneal scrape from the edge of the epithelial defect was performed. The culture showed no growth. The patient was commenced on oral fluconazole $200 \mathrm{mg}$ $\mathrm{BD}$, G. penicillin, G. gentamicin and G clotrimazole one

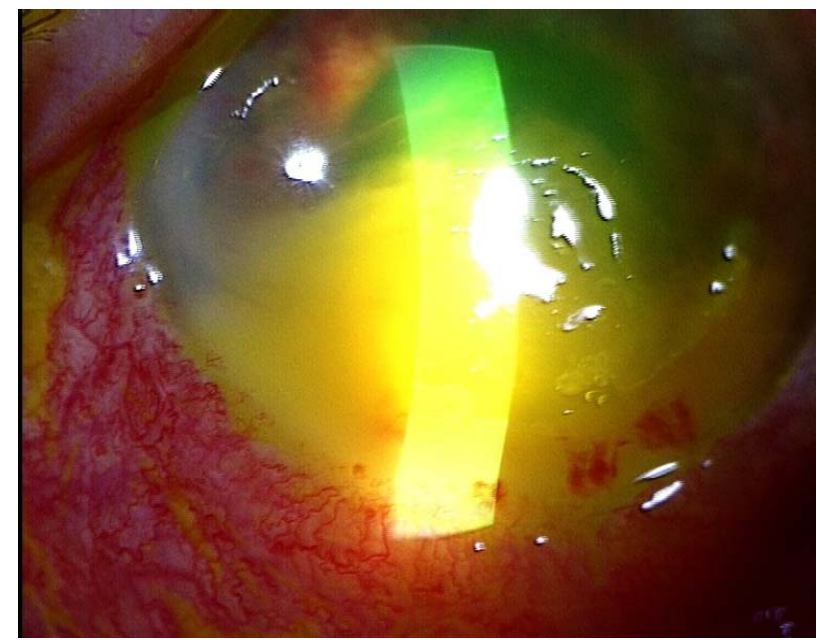

Figure 1. Left eye hemorrhagic hypopyon on presentation. 
hourly day and night. As the corneal signs improved, with a reduction in the epithelial defect and hypopyon, the patient was discharged. Unfortunately, on follow up in clinic, there was an increase in the hypopyon and epithelial defect with an anterior stromal infiltrate. The patient was readmitted and a corneal biopsy and anterior chamber washout was performed. Histology showed an acute inflammatory exudate permeating the corneal stroma and multiple septate and branching fungal hyphae with spores.

Culture identified cylindrocarpon species morphologically resembling Cylindrocarpon destructans (mycology reference lab Bristol, UK).

The patients treatment was changed to voriconazole $200 \mathrm{mg}$ BD, G. amphotericin and G. clotrimazole one hourly. This resulted in a reduction in symptoms, hypopyon and infiltrate and so, the patient was discharged home. However, three day following discharge, VA was hand movements and corneal perforation with a flat anterior chamber was noted. An urgent tectonic penetrating keratoplasty (PKP) was performed. Postoperative treatment included G. chloramphenicol 2 hourly, G. Natamycin six times a day, G. Atropine OD, G. Dexemathasone preservative free QDS, G. Ciprofloxacin TDS and oral voriconazole $200 \mathrm{mg}$ BD.

Five week following the PKP, the graft remained clear and all treatment was stopped except dexamethasone, chloramphenicol and natamycin QDS. Two month following the PKP, the patient presented to clinic complaining of a white spot on the left eye. Examination revealed a $1.2 \mathrm{~mm} \times 1.2 \mathrm{~mm}$ epithelial defect with a 5.5 mm hypopyon (Figure 2). Initially, the chloramphenicol and dexamethasone were reduced to TDS, natamycin was increased to 2 hourly and the patient was recommenced on oral voriconazole $200 \mathrm{mg}$ BD. Three days later, the hypopyon had reduced but a deep stromal infiltrate was noted at the junction of the graft and host. Topical dexamethasone was stopped and in addition to the above treatment, oral ciprofloxacin and flucloxacillin were added. One week later, the stromal infiltrate had increased. We therefore performed an anterior chamber washout during which we noted thick exudate adherent to the corneal endothelium and iris with significant peripheral synechiae. Intracameral amphoteracin and vancomycin were administered. Post operatively, the patients treatment was simplified to oral Voriconazole 200 mg BD and G. amphoteracin $0.15 \%$ two hourly. On initial follow-up, the stromal infiltrate had reduced.

Two week following treatment the infiltrate had further reduced, however, there was superior superficial vascularisation of the corneal graft, a persistent epithelial defect, and a descemetocoele (siedel negative) (Figure $3)$.

The case was discussed at our regional corneal grand

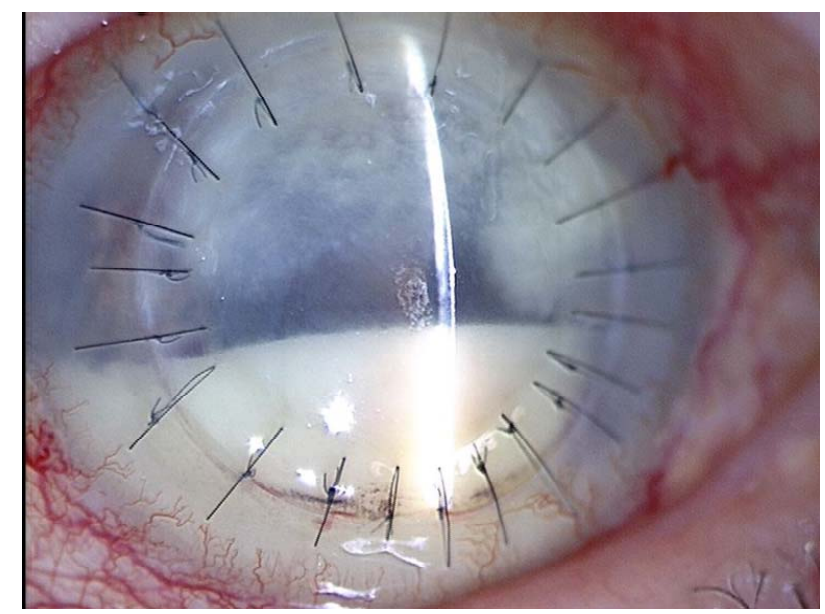

Figure 2. Left eye two month following PKP. Hypopyon shown.

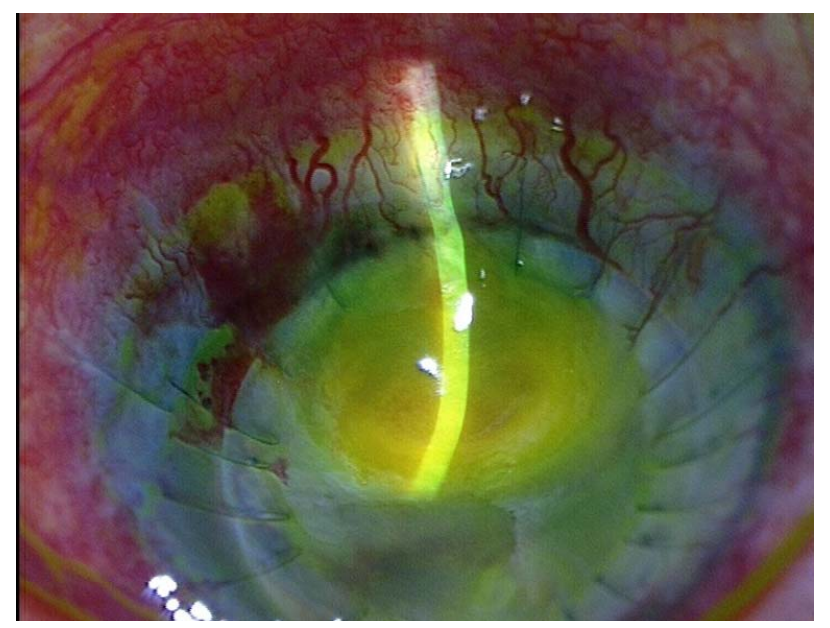

Figure 3. Left eye superficial vascularisation, epithelial defect and a descemetocoele.

round and the consensus was that the patient is likely to have significant anterior segment scarring with a poor prognosis with any further surgical intervention.

On six month follow up, the epithelial defect had healed and all treatment was stopped. The patient has not had any further recurrences.

\section{Discussion}

We present the first reported case of Cylindrocarpon destructans causing keratitis in humans. Furthermore, the case highlights the dilemma of the duration of antifungal treatment both in the primary infection and following penetrating keratoplasty (PKP) and the risks of using of topical steroids following PKP.

Cylindrocarpon destructans is a soil fungus often associated with plant roots. They may be saprobes or facultative plant pathogens.

To date there have been several reported cases of Cyl- 
indrocarpon lichenicola causing keratitis in humans [1-4] and a single case series of Cylindrocarpon destructans causing keratitis in horses [5]. However, the only reported case of Cylindrocarpon destructans causing disease in humans is that of a chronic foot abscess in a 39 year old male [6].

There is very little clinical evidence on the use or timing of use of topical steroids in the medical management of PKP in patients with fungal keratitis. The clinical dilemma with the post-operative use of topical steroids is reduction of inflammation \& prevention of rejection versus the risk of recurrence of fungal keratitis.

In a series of 54 patients with fungal keratitis who underwent PKP [7], patients were divided into 2 post-operative treatment arms. Group one received topical fluconazole (doflucan) and topical ciclosporin and group two received only topical fluconazole. The authors found that there was no increase in the recurrence rate of fungal keratitis in-group one compared to two and, the eyes were less inflamed in group one. Hence, there may be a role for the use of topical ciclosporin post PKP in this group of patients.

With regards to the use of topical steroids following PKP, in a large series of 358 patients with fungal keratitis [8], systemic and topical steroids (specific drug names were not stated) as well as topical $1 \%$ ciclosporin were given 2 weeks after PKP if recurrence did not occur. The authors choose 2 weeks as all the recurrences $(7.4 \%$ of PKP performed) occurred within 2 weeks post operatively.

There is very little information in the literature on the duration of anti-fungal treatment. In the above mentioned series [8], both topical $(0.25 \%$ amphotericin and $5 \%$ natamycin) and an oral fluconazole were used. However, only the duration of topical antifungal treatment was stated (five days to one month (mean eleven days)). In those patients that required emergency PKP (108\% $32 \%$ ), during the procedure, the hypopyon $\&$ fibrinous membrane were carefully removed and the anterior chamber was irrigated with fluconazole $0.2 \%$. Post operatively, subconjunctival fluconazole was given for 3 days, oral fluconazole was given for 21 days, fluconazole or amphotericin B ointment was administered at night and fluconazole 1\% drops QID were given during the day. All treatment was tapered and stopped by day 20 - 30 post-operatively. Our case is unusual in that recurrence occurred two months following PKP.

In conclusion, this is the first reported case of Cylindrocarpon destructans causing keratitis in humans. Furthermore, the case highlights the contentious issues of the use of topical steroids following PKP, the potential role of topical ciclosporin and the duration of antifungal treatment both in primary infection and following PKP. Antifungal treatment may need to be continued for several months, particularly following PKP.

\section{REFERENCES}

[1] C. Booth, Y. M. Clayton and M. Usherwood, "Cylindrocarpon Species Associated with Mycotic Keratitis,” Proceedings of the Indian Academy of Sciences, Plant Sciences, Vol. 94, No. 2, 1985, pp. 433-436.

[2] S. Laverde, L. H. Moncada, A. Restrepo, et al., "Mycotic Keratitis: 5 Cases Caused by Unusual Fungi,” Sabouraudia, Vol. 11, No. 2, 1973, pp. 119-123.

[3] T. Matsumoto, J. Masaki and T. Okabe, "Cylindrocarpon Tonkinense: As a Cause of Keratomycosis,” Transactions of the British Mycological Society, Vol. 72, 1979, pp. 503504.

[4] A. Mitra, V. Savant, A. Aralikatti, et al., "The Use of Voriconazole in the Treatment of Cylindrocarpon Keratomycosis," Cornea, Vol. 28, No. 2, 2009, pp. 217-218. doi:10.1097/ICO.0b013e3181870315

[5] N. T. Chmielewski, P. J. Smith, D. E. Brooks, et al., "Keratomycosis in Four Horses Caused by Cylindrocarpon Destructans," Veterinary \& Comparative Ophthalmology, Vol. 6, No. 4, 1996, pp. 252-256.

[6] D. E. Zoutman and L. Sigler, "Mycetoma of the Foot Caused by Cylindrocarpon Destructans," Journal of clinical Microbiology, Vol. 29, No. 9, 1991, pp. 1855-1859.

[7] G. J. Wu, W. Ding and F. Y. Yang, "Administration of Immunosuppressive Drug and Corticosteroid after Penetrating Keratoplasty in Fungal Corneal Ulcer,” Chinese Ophthalmic Research, Vol. 19, No. 1, 2001, pp. 64-66.

[8] L. X. Xie, X. G. Dong and W. Y. Shi, "Treatment of Fungal Keratitis by Penetrating Keratoplasty,” British Journal of Ophthalmology, Vol. 85, No. 9, 2001, pp. 10701074. doi:10.1136/bjo.85.9.1070 